\title{
Projection, Entanglement and Nonlocality of Photon-Number Entangled States Generated in Kerr Media
}

\author{
M. NowOtARski ${ }^{a, *}$ AND J.K. KALAGA ${ }^{a, b}$ \\ ${ }^{a}$ Quantum Optics and Engineering Division, Institute of Physics, University of Zielona Góra, \\ Prof. Z. Szafrana 4a, 65-516 Zielona Góra, Poland \\ ${ }^{b}$ Joint Laboratory of Optics of Palacký University and Institute of Physics of CAS, \\ Faculty of Science, Palacký University, 17. listopadu 12, 77146 Olomouc, Czech Republic
}

Doi: 10.12693/APhysPolA.139.610

*e-mail: m.nowotarski@if.uz.zgora.pl

\begin{abstract}
Motivated by the proposal for quantification of Bell nonlocality Phys. Rev. A 92, 030101 (2015), and the concept of ICPS symmetric states Phys. Rev. A 95, 042333 (2017), we present applications of those tools in the case of the physical system, namely photon-number entangled states generated in Kerr media with optical parametric pumping Prog. Opt. 41, 361 (2000). We start from the projection of a density matrix of the considered physical system to the family of ICPS states. For each step of the considered time evolution, we subsequently calculate both the negativity and negativity of projections to examine the lower bound of entanglement versus the actual one. Then we use those projections to calculate the lower bound of nonlocality for the volume of violation.
\end{abstract}

topics: nonlinear Kerr oscillators, entanglement, nonlocality

\section{Introduction}

Quantum correlations play a crucial role in many practical applications of quantum mechanics. They are used in quantum teleportation protocols, quantum computation algorithms, and quantum cryptography, to name just a few. For that reason, much effort has been put into the development of quantum correlation measures such as measures of quantum entanglement and quantum nonlocality. As it was discussed in [1] (and later continued in [2, 3]), there exist states that, although not maximally entangled, maximally violate a given Bell-type inequality. Such an anomaly was an inspiration for the development of a new type of quantum nonlocality measures, namely, the volume of violation [4]. This innovative approach changed the way we quantify nonlocality.

In this paper, we study a model of two nonlinear Kerr oscillators mutually coupled by parametric pumping described in [5]. For such a system, we calculate a quantum measure of entanglement, namely, the negativity. Next, we project the time evolution of the density matrix of the given state into the family of incomplete-permutation-symmetric states (ICPS) [6]. For such a time evolution of projected states, we again calculate the negativity and then compare all those results with the volume of violation to show the relation between them.

\section{Model}

We consider a system composed of two nonlinear Kerr oscillators mutually coupled by parametric pumping. In the given system, pairs of photons via spontaneous parametric down-conversion are produced. The Hamiltonian describing the analyzed system takes the form [5]

$$
\begin{aligned}
& \hat{H}_{\mathrm{int}}=\frac{\chi_{a}}{2}\left(\hat{a}^{\dagger}\right)^{2} \hat{a}^{2}+\frac{\chi_{b}}{2}\left(\hat{b}^{\dagger}\right)^{2} \hat{b}^{2}+G \hat{a}^{\dagger} \hat{a} \hat{b}^{\dagger} \hat{b} \\
& +g \hat{a}^{\dagger} \hat{b}^{\dagger}+\bar{g} \hat{a} \hat{b} .
\end{aligned}
$$

The first two terms of the Hamiltonian describe the nonlinear Kerr-type oscillators characterized by the nonlinearity constants $\chi_{a}$ and $\chi_{b}$. The next term is the Kerr cross term, whereas the last two ones are related to the two-mode parametric process. Parameter $g$ represents the strength of the external field. Operators $\hat{a}^{\dagger}\left(\hat{b}^{\dagger}\right)$ and $\hat{a}(\hat{b})$ are the creation and annihilation operators, respectively, related to the subsystems $A(B)$.

The assumptions used in the analysis are: the strength of the external field $g=0.6$, the nonlinearities $\chi_{a}=\chi_{b}=1$, and the nonlinear coupling constant $G=2 \chi_{a}$. Moreover, the initial state is set to $|\psi(t=0)\rangle=|0\rangle_{a}|0\rangle_{b}$. The fact that our system starts from the vacuum state and $g<\chi_{a}+\chi_{b}$ practically limits the time evolution to three possible states. Thus, the truncated wave function can be expressed in the following form: 


$$
|\psi(t)\rangle_{\text {cut }}=c_{00}(t)|00\rangle+c_{11}(t)|11\rangle+c_{22}(t)|22\rangle
$$
where $\left|c_{i i}\right|$ stands for the probability of finding our system in state $|i i\rangle$. To show to what degree the trunctuation is accurate, in Fig. 1 we present the function $1-F(t)$. Here,

$$
F(t)=\left|c_{00}\right|^{2}+\left|c_{11}\right|^{2}+\left|c_{22}\right|^{2}
$$

and is calculated for the "full" wave function obtained from numerical analysis. In this way, we determine whether or not we have truncated probabilistically significant states from the "full" wave function. In Fig. 1, we can see that the deviation of the function $F(t)$ from unity is of the order of $10^{-5}$, and hence, the states with a higher number can be neglected.

\section{Projection}

In our study, we want to compare the generation of entangled states in the evolution of our system with the entanglement production of the projection onto the recently introduced ICPS states [6]. The ICPS is a family parametrized by five real numbers that, in general, describes mixed states with a property of high symmetry. The operation of projection (often termed twirling) was introduced in [7]. Importantly, this operation cannot increase the degree of entanglement because local unitary operations cannot increase the entanglement either [8].

Let $\rho(t)=|\psi(t)\rangle_{\text {cut }} \otimes_{\text {cut }}\langle\psi(t)|$. The density matrix $\tilde{\rho}(t)$ of the projected state $\mathbb{P}: \rho \rightarrow \tilde{\rho}$ takes the form:

$$
\tilde{\rho}(t)=\left[\begin{array}{ccccccccc}
a_{1} & 0 & 0 & 0 & b_{1} & 0 & 0 & 0 & b_{2} \\
0 & 0 & 0 & 0 & 0 & 0 & 0 & 0 & 0 \\
0 & 0 & 0 & 0 & 0 & 0 & 0 & 0 & 0 \\
0 & 0 & 0 & 0 & 0 & 0 & 0 & 0 & 0 \\
b_{1} & 0 & 0 & 0 & a_{1} & 0 & 0 & 0 & b_{2} \\
0 & 0 & 0 & 0 & 0 & 0 & 0 & 0 & 0 \\
0 & 0 & 0 & 0 & 0 & 0 & 0 & 0 & 0 \\
0 & 0 & 0 & 0 & 0 & 0 & 0 & 0 & 0 \\
b_{2} & 0 & 0 & 0 & b_{2} & 0 & 0 & 0 & a_{4}
\end{array}\right],
$$

where $a_{1}=\frac{1}{2}\left(\left|c_{00}\right|^{2}+\left|c_{11}\right|^{2}\right), a_{4}=1-2 a_{1}$, $b_{1}=\operatorname{Re}\left(\overline{c_{00} c_{11}}\right), \quad b_{2}=\operatorname{Re}\left(\overline{c_{00} c_{22}}\right)+\operatorname{Re}\left(\overline{c_{11} c_{22}}\right)$. The time evolution of the projection parameters corresponding to the density matrix $\tilde{\rho}(t)$ is presented in Fig. 2. We can see that the two parameters most fluctuating in time are $b_{1}$ and $b_{2}$, which are related to off-diagonal elements of the density matrix $\tilde{\rho}(t)$.

\section{Entanglement and volume of violation}

To quantify the amount of entanglement generated in our system, we used a well-established measure of entanglement called negativity [9-11]. In the most general scenario, negativity is defined as:

$$
N(\rho)=\left(\left\|\rho^{T_{A}}\right\|_{1}-1\right) / 2,
$$

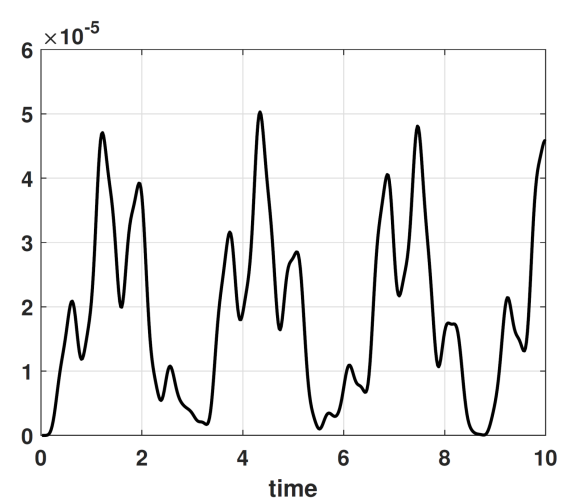

Fig. 1. Time evolution of the $1-F(t)$ function for $|\psi(t=0)\rangle=|0\rangle_{a}|0\rangle_{b}, \chi_{a}=\chi_{b}=1$, and $g=0.6$. Time is scaled in the units of $1 / \chi_{a, b}$.

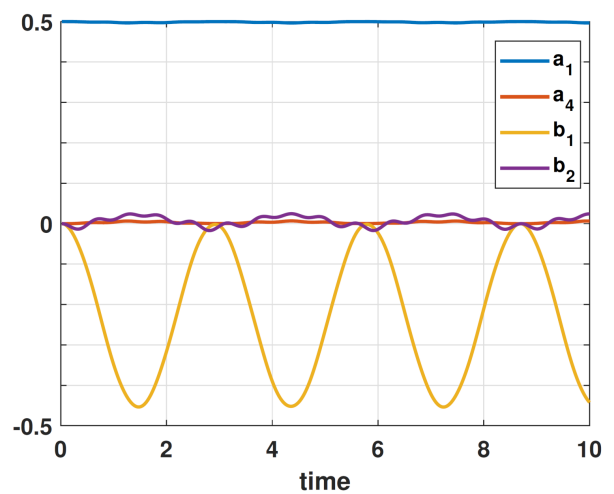

Fig. 2. Time evolution of $a_{1}, a_{4}, b_{1}, b_{2}$ as elements of projected density matrix $\tilde{\rho}(t)$. The values of parameters are the same as for Fig. 1.

where $\|\cdots\|_{1}$ means the trace norm of the matrix (sum of negative eigenvalues), and $\rho^{T_{A}}$ is a partially transposed density matrix. Such a formula is universal for all density matrices, and we applied it to calculate the time evolution of the negativity of the states described in (2).

Next, we used the formula from [6] to calculate the negativity for projections $\tilde{\rho}(t)$, namely,

$$
N(\tilde{\rho}(t))=\left|b_{1}\right|+2\left|b_{2}\right| .
$$

The time evolution of both negativities, that is, $N(\rho(t))$ and $N(\tilde{\rho}(t))$, is illustrated in Fig. 3. The analyzed period is large enough to observe a periodical behavior in the amount of entanglement generation. Initially starting from zero, the negativity $N(\rho(t))$ quite fast breaks the limit of 0.3 and converges to its maximum around 0.55 .

The same type of behavior can be observed for $N(\tilde{\rho}(t))$. What is noticeable, the negativity of the projected states is always lower than the actual negativity of $\rho$. It is in line with the statement that projections cannot increase the degree of entanglement. Thus, the projections always give a lower bound of the entanglement properties. In this scenario, we can see that $N(\tilde{\rho}(t))$ follows quite accurately the periodical change of $N(\rho(t))$. The mean 


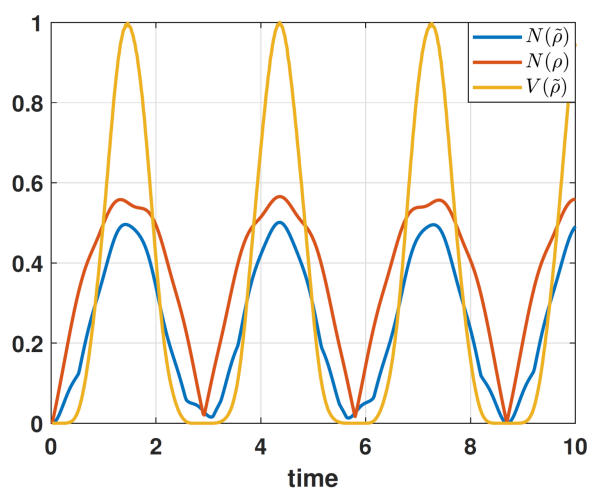

Fig. 3. Time evolution of $\mathcal{N}(\rho), \mathcal{N}(\tilde{\rho})$ and $V(\rho)$. Volume of violation was normalized. The values of parameters are the same as for Figs. 1 and 2.

value of the difference $N(\rho(t))-N(\tilde{\rho}(t))$ is 0.1218 , which is around $25 \%$ of the mean value of $N(\rho(t))$. It is because the ICPS state family, although simple and symmetric, is quite wide. On the other hand, the system described by (2) is quite simple and thus close to the ICPS state.

Finally, we use the recently introduced measure of nonlocality, namely, the volume of violation [4], to calculate the nonlocality of the projected states $\tilde{\rho}(t)$. This measure is defined as

$$
V(\rho, I)=\int_{\Gamma_{\rho, I}} \mathrm{~d}^{n} x,
$$

where $\mathrm{d}^{n} x=\mathrm{d} x_{1} \ldots \mathrm{d} x_{n}$, and $\Gamma_{\rho, I} \subset X$ is the subset of all possible experimental configurations that lead to the violation of a given Bell-type inequality $I$.

The idea of this measure is to take into account how often a given Bell-type inequality is violated in a given experimental scenario. This is different from the usual approach when we take into account how much a given inequality has been violated. We use then CGLMP inequality [12] and the set of local observables $M_{1}$ (more details in [13]). The normalized evolution of $V$ with respect to time has been presented in Fig. 3 together with entanglement measures. We can observe the same periodicity as in the case of $N(\rho(t))$ and $N(\tilde{\rho}(t))$. The evolution of $V$ is more polarized in the sense that there is a longer time where there is no nonlocality detected. This is contrary to entanglement which, although often small, is detected almost all the time.

\section{Conclusion}

In the present paper, the model of two nonlinear Kerr oscillators mutually coupled by parametric pumping was considered. We have used this model to present the application of two main concepts. First, we projected the time evolution of the density matrix of our model onto the family of ICPS states using the projection operator $\mathbb{P}$. For projected and normal (before projection) states, we have calculated the amount of generated entanglement using negativity. The goal was to compare those two values with each other to see how much accuracy, in that specific scenario, we are losing when projecting states. Next, we used the volume of violation to calculate the amount of nonlocality in a different than usual approach. We compared those results with entanglement. The key idea was to present the possibilities of those two methods: projection $\mathbb{P}$ and nonlocality $V$ on a simple model and analyze how it works.

In more complicated scenarios, when the density matrix of the quantum system could be much less sparse, those methods could give a grasp idea about entanglement and nonlocality properties, which lower the computational cost of using projection states.

\section{Acknowledgments}

M.N. and J.K.K. acknowledge the support of the program of the Polish Minister of Science and Higher Education under the name "Regional Initiative of Excellence" in 2019-2022, project No. 003/RID/2018/19, funding amount 11936 596.10 PLN. J.K.K. acknowledges the support by the ERDF/ESF project "Nanotechnologies for Future" (CZ.02.1.01/0.0/0.0/16_019/0000754).

\section{References}

[1] A. Acín, T. Durt, N. Gisin, J.I. Latorre, Phys. Rev. A 65, 052325 (2002).

[2] S. Zohrenm, R.D. Gill, Phys. Rev. Lett. 100, 120406 (2008).

[3] J.-L. Chen, C. Wu, L.C. Kwek, C.H. Oh, M.-L. Ge, Phys. Rev. A 74, 032106 (2006).

[4] E.A. Fonseca, F. Parisio, Phys. Rev. A 92 , 030101 (2015).

[5] J. Peřina Jr., J. Peřina, Prog. Opt. 41, 361 (2000).

[6] A. Barasiński, M. Nowotarski, Phys. Rev. A 95, 042333 (2017).

[7] K.G.H. Vollbrecht, R.F. Werner, Phys. Rev. A 64, 062307 (2001).

[8] C. Eltschka, G. Tóth, J. Siewert, Phys. Rev. A 91, 032327 (2015).

[9] K. Życzkowski, P. Horodecki, A. Sanpera, M. Lewenstein, Phys. Rev. A 58, 883 (1998).

[10] G. Vidal, R.F. Werner, Phys. Rev. A 65, 032314 (2002).

[11] A. Peres, Phys. Rev. Lett 77, 1413 (1996).

[12] D. Collins, N. Gisin, N. Linden, S. Massar, S. Popescu, Phys. Rev. Lett. 88, 040404 , (2002).

[13] A. Barasiński, M. Nowotarski, Phys. Rev. A 98, 022132 (2018). 